\title{
Atomistic simulations of tensile deformation in a CrCoNi medium-entropy alloy with heterogeneous grain structures
}

\author{
Fuping Yuan ${ }^{\mathrm{a}, \mathrm{b}, *}$, Wenqiang Cheng ${ }^{\mathrm{a}, \mathrm{b}}$, Shengde Zhang ${ }^{\mathrm{a}, \mathrm{b}}$, Xiaoming Liu ${ }^{\mathrm{a}, \mathrm{b}}$, Xiaolei Wu ${ }^{\mathrm{a}, \mathrm{b}}$ \\ ${ }^{a}$ State Key Laboratory of Nonlinear Mechanics, Institute of Mechanics, Chinese Academy of Science, 15 Beisihuan West Road, Beijing 100190, China \\ ${ }^{\mathrm{b}}$ School of Engineering Science, University of Chinese Academy of Sciences, 19A Yuquan road, Beijing 100049, China
}

\section{A R T I C L E I N F O}

\section{Keywords:}

High-entropy alloys

Strain partitioning

Phase transformation

Plastic deformation mechanisms

Twinning

Molecular dynamics simulation

\begin{abstract}
A B S T R A C T
Large-scale molecular dynamics simulations have been applied to investigate the atomistic deformation mechanisms of tensile deformation in a CrCoNi medium-entropy alloy with heterogeneous grain structures. After yielding, the heterogeneous grain structures show strong strain hardening due to grain-to-grain yielding and Masing hardening. The grain-to-grain yielding can be attributed to the various grain sizes and the different Schmidt factors. The HCP transformation and formation of hierarchical deformation nanotwins have been observed in the CrCoNi with heterogeneous grain structures due to the low stacking fault energy at cryogenic temperature. The HCP phase was found to be formed by simultaneous nucleation and propagation of intrinsic stacking faults at adjacent slip planes from grain boundaries (GBs). It was found that there is no equivalent strain partitioning between large grains and small grains in the heterogeneous grain structures, which can be attributed to that GBs of small grains can also accommodate significant shear strains due to the enhanced GB activities for small grains. While tensile strain partitioning between large grains and small grains in the heterogeneous grain structures was observed, and this tensile strain partitioning was found to become more obvious with increasing grain size ratio between large grains and small grains. The simulation results should provide insights of designing heterogeneous structures for achieving better mechanical properties.
\end{abstract}

\section{Introduction}

High-entropy alloys (HEA) [1-19] and/or medium-entropy alloys (MEA) [20-30], consisting of three or more elements generally with equal molar fraction, have emerged recently as an attractive class of materials due to their superior mechanical properties. The energy penalty for the formation of stacking fault (SF), i.e. the SF energy (SFE) in this class of alloys especially with FCC crystal structure, is typically low due to the random distribution of multiple elements [3,4,6,20,22,24$27,29,30]$. Ab initio modeling even suggested that the CrCoNi MEA can have a negative SFE at $0 \mathrm{~K}$ and high propensity for twinning [22]. Recent work has also shown that the SFE could be non-uniform and tunable locally in this kind of alloys by tailoring local chemical order [31]. Moreover, the FCC HEAs and MEAs, such as FeCrMnCoNi HEA [3,4,6] and CrCoNi MEA [20,22,24-27,29,30], have better tensile and fracture properties at cryogenic temperature than at room temperature. The better mechanical properties at cryogenic temperature can be attributed to a transition of dominant deformation mechanism from dislocation slip to deformation twinning $[4,20]$.
Metals and alloys with coarse grains (CG) generally have a low yield strength, and the yield strength can be significantly elevated by refining grain size to nanoscale, while accompanied with obvious sacrifice of ductility $[32,33]$. Typically, the strength and the ductility are mutually exclusive, a trade-off exists in metals and alloys with homogeneous structures [32-34]. Severe plastic deformation followed by critical annealing has been found to produce a heterogeneous grain structure in metals and alloys, resulting in a better synergy of strength and ductility $[25,26,35-38]$. In such heterogeneous structure, strain gradients can be induced at the domain boundaries due to the stress/strain partitioning between various domains with dramatically different mechanical properties, resulting in strong back stress hardening/hetero-deformation induced hardening for better tensile properties [36,39-41]. This strategy has also been utilized in HEAs and MEAs $[25,26,30]$ to produce superior quasi-static tensile properties or excellent dynamic properties at both cryogenic and room temperatures.

Atomistic simulations have been proven to have advantages for investigating the deformation behaviors and atomistic deformation mechanisms in metals and alloys with carefully designed simulation

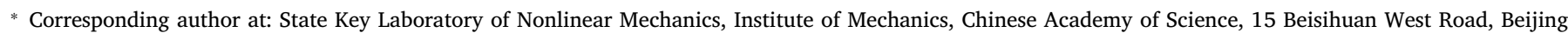
100190, China..

E-mail addresses: fpyuan@lnm.imech.ac.cn (F. Yuan), vestentceng@qq.com (W. Cheng), zhangshengde@imech.ac.cn (S. Zhang), xiaomingliu@lnm.imech.ac.cn (X. Liu), xlwu@imech.ac.cn (X. Wu).
} 

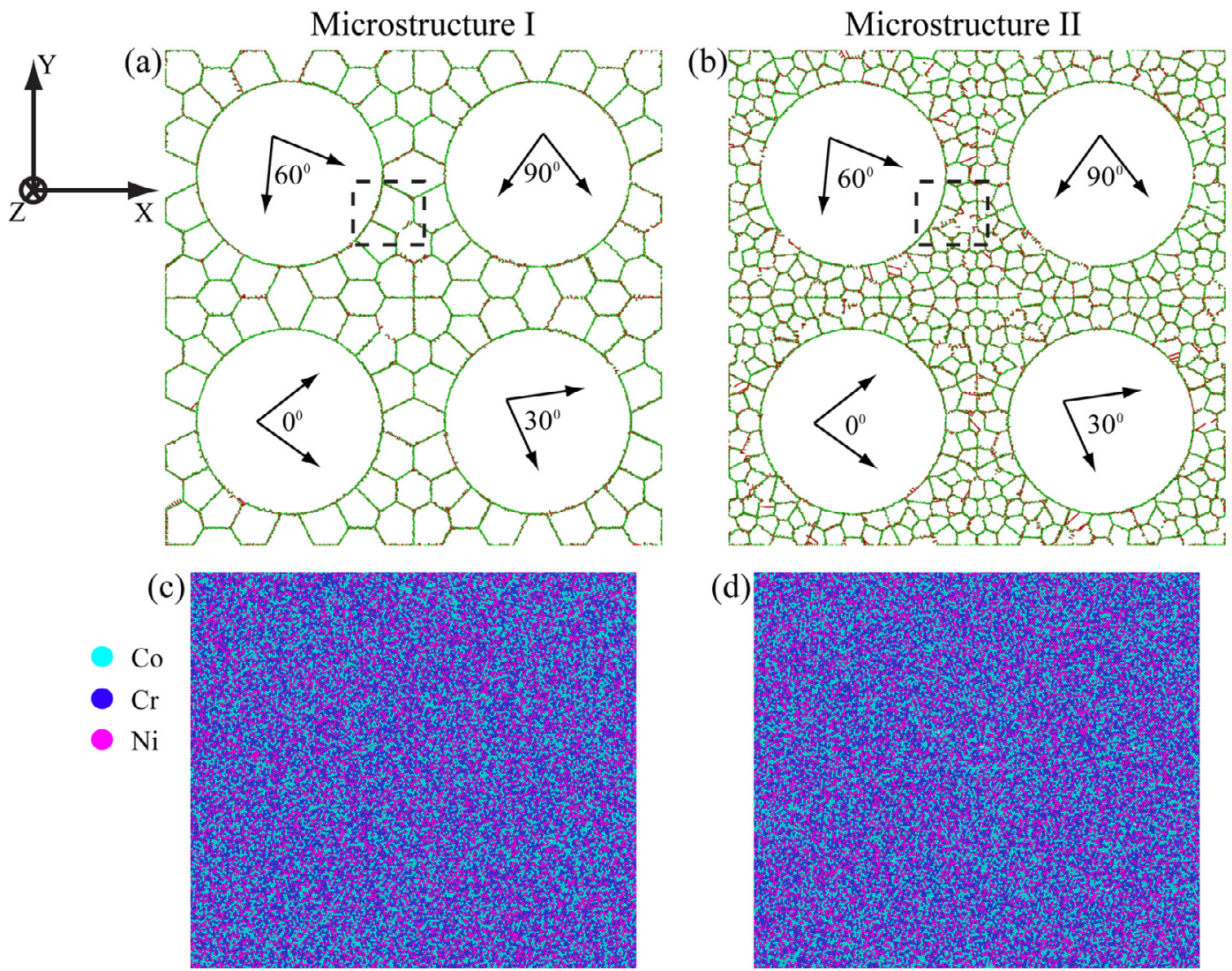

Fig. 1. The relaxed simulation cells for two different heterogeneous grain structures: I (a) and II (b). The distributions for Co, $\mathrm{Cr}$ and Ni atoms in the areas marked by the rectangular in (a) and (b) are displayed in (c) and (d).

models, in which the real-time evolutions of the microstructures (snapshots) can be provided [42,43]. Molecular dynamics (MD) simulations have also been utilized to investigate the atomistic deformation mechanisms in HEAs and MEAs [44-54]. For example, MD simulations have revealed that the $\mathrm{Al}_{0.3} \mathrm{CoCrFeNi}$ HEA with embedded $\mathrm{B} 2$ grains can induce thicker deformation twins by enhancing deformation twinning due to the raised local stress level, as compared to the single fcc-phase condition [45]. The uniaxial tensile behaviors and nanoscratching behaviors in a AlCrCuFeNi HEA have been studied using MD simulations $[46,47]$. A hydrid Monte Carlo/MD method has also been used to evaluate the temperature-dependent chemical order in a MoNbTaW HEA with bcc structure [48]. MD simulations have shown transformation induced softening and plasticity in a CoNiFeAlCu HEA [49]. For FeCrMnCoNi HEA and CrCoNi MEA, several previous efforts have studied the grain size effects [50], dislocation behaviors [51], nanoindentation behaviors [52], alloying effect and micro-twinning behaviors [53] by utilizing MD simulations. In these studies, the simulation models were single crystal, bi-crystal and polycrystal with nearly homogeneous grain size [44-54]. Here, we advocated a different perspective: the tensile deformation behaviors in the CrCoNi MEA with heterogeneous grain structures were investigated, and the revealed atomistic deformation mechanisms might provide insights for the previously observed experimental phenomenon $[25,26,30]$ and help designing heterogeneous structures to achieve better mechanical properties.

\section{Simulation techniques}

The atomistic simulations have been conducted by the open source Large-scale Atomic/Molecular Massively Parallel Simulator (LAMMPS) [55] and the force reaction between atoms was simulated by a EAM potential developed by Farkas [56]. In this study, two heterogeneous grain structures were considered, in which the grain sizes of large grains were both set to be $120 \mathrm{~nm}$, while the average grain sizes of small grains were 23.44 and $11.5 \mathrm{~nm}$, respectively, in these two heterogeneous grain structures (Microstructures I and II). Thus, the grain size ratios between the large grains and the small grains were 5.1 and 10.4, respectively, for these two heterogeneous grain structures. Since the considered grain sizes (11.5 nm to $120 \mathrm{~nm}$ ) were larger than those possible in fully 3dimensional simulations, thus [110]-textured simulation cells (similar to configurations used in previous research [57]) with columnar grains were considered in the present study. The two simulation cells for the two different heterogeneous grain structures are displayed in Fig. 1(a) and (b). The relative misorientation angles for four large grains $\left(0^{\circ}\right.$, $30^{\circ}, 60^{\circ}, 90^{\circ}$ are selected to cover various Schmidt factors to minimize the orientation effect) and the corresponding $\{111\}$ slip planes are also marked in the Fig. 1(a) and (b). Microstructures I and II contain 104 and 432 small grains with random orientation angles, respectively. The simulation cells contain 12 layer atoms along $z$ direction, the sizes of simulation cells for two microstructures are both $320 \times 320 \times 1.51 \mathrm{~nm}^{3}$, and the simulation cells contain approximately $14,000,000$ atoms. Fig. 1 (c) and (d) display the distributions for $\mathrm{Co}, \mathrm{Cr}$ and $\mathrm{Ni}$ atoms in the areas marked by the rectangular in Fig. 1(a) and (b). It is clearly indicated that three atoms have random distributions in the simulation cells. As indicated in Fig. 1(a) and (b), atoms are colored based on common neighbor analysis (CNA) values in order to identify defects (red color for HCP atoms, green color for dislocation core and grain boundary (GB) atoms, gray color for perfect FCC atoms which are not shown in Fig. 1(a) and (b)).

Periodic boundary conditions were imposed for all three directions during simulations. The as-created samples were first subjected to energy minimization by the conjugate gradient method, and then gradually heated up to $77 \mathrm{~K}$ and finally relaxed under both 0 bar pressure in all three directions and the temperature $77 \mathrm{~K}$ for enough time to reach equilibrium using the Nose/Hoover isobaric-isothermal ensemble. Uniaxial tensile loading were imposed on the relaxed samples along $x$ direction 


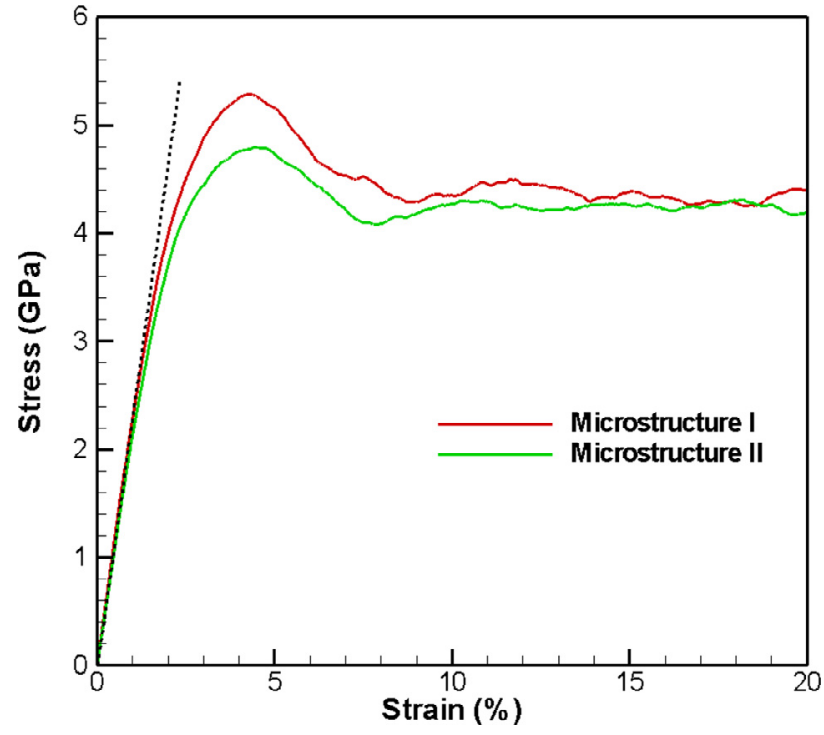

Fig. 2. Simulated stress strain curves for the two heterogeneous grain structures.

with a constant strain rate of $5 \times 10^{8} \mathrm{~s}^{-1}$ to a total tensile true strain of $20 \%$ by keeping the pressures in the other two directions to be zero during loading. In order to check the strain partitioning between the large grains and the small grains, the atomic local strain tensor was computed as a best fit of the local affine transformation, and was obtained by running a least-square fitting procedure using all the local neighbor atom's information with a cut-off distance $0.5 \mathrm{~nm}[58,59]$. Thus, the tensile strain $\varepsilon_{11}$ for each atom can be obtained from the local strain tensor $\tilde{\varepsilon}$, and the local equivalent strain for each atom can be calculated by the local strain tensor as following:

$\varepsilon_{e q}=\sqrt{J_{2}(\tilde{\varepsilon})}$

where $J_{2}(\tilde{\varepsilon})$ is the second deviatoric strain invariant.

\section{Results and discussions}

\subsection{Stress strain curves and Masing hardening}

The simulated stress strain curves for two heterogeneous microstructures are shown in Fig. 2, in which the elastic regime is marked by a straight dash line. After elastic stage, the stress strain curves start to deviate from the linear relationship, and show a strong strain hardening in a short strain range. Then, the stresses start to decrease gradually to a plateau with small fluctuations. These simulated stress-strain behaviors have discrepancies with those found in the experiments [25,30], where the stress-strain curves harden over a much larger range of strain and the maximum stress is reached very close to the fracture strain where necking sets in. These discrepancies can be attributed to two points: (i) the stress overshoot phenomenon induced by the high strain rate employed in MD simulations [43,45,49]; (ii) the average grain size employed in the present study is much smaller compared to those for the experiments $[25,30]$, less strain hardening in a shorter strain range should be observed for the polycrystal samples with nanocrystalline grains when the necking is suppressed by the lateral periodic boundary conditions instead of free boundary conditions (as described for the present simulations). The microstructure I shows a higher maximum strength and a slightly higher plateau value of flow stress when compared to those for microstructure II. It should be noted that the average grain sizes of small grains were 23.44 and $11.5 \mathrm{~nm}$ in the microstructure I and the microstructure II, respectively. Previous research have shown that the strength starts to decrease with further grain refinement below the critical value $(\sim 15 \mathrm{~nm})$ for fcc metals [42], suggesting a shift in deformation mechanisms from dislocation activities to GB activities. Thus, the lower flow stress in the microstructure II should be due to the GB-mediated deformation softening mechanisms. In order to understand the strong strain hardening after the elastic stage, and the snapshots of atomistic structures and the equivalent strain contours for microstructure I at applied tensile strains of $2 \%, 3 \%$ and $4 \%$ are displayed in Fig. 3. It can be clearly observed that defects, such as dislocations and SFs, are only generated in two large grains and in part of small grains at the applied tensile strain of $2 \%$. These indicate that the other two large grains and most small grains are still in elastic deformation at the applied tensile strain of $2 \%$, which can also be identified by the equivalent strain contour in Fig. 3(d) in which most grains are in blue color. It also should be noted that the equivalent strains at defects, such as SFs, dislocation cores and GBs, are much higher compared to the other areas. It is easy to understand since the defects are the main carriers of plastic strain. With increasing applied tensile strain, the number of grains with defects and under plastic deformation increases, and almost all grains are under plastic deformation at the applied tensile strain of $4 \%$ (with exceptions for a few grains).

These observations indicate that the yielding in the samples is grainto-grain due to the various grain sizes and the various Schmidt factors for each grain. The grain-to-grain yielding here means that most grains yield one by one in a sequence with increasing applied tensile strain. Thus, the strong strain hardening stage after yielding should correspond to the elasto-plastic transition stage, and the strong strain hardening should be attributed to the Masing hardening [60]. It has been indicated that load transfer and plastic strain partitioning between hard domains (small grains and/or grains with small Schmidt factors) and soft domains (large grains and/or grains with large Schmidt factors) should occur during the elasto-plastic transition stage, and the back stress hardening/ hetero-deformation induced hardening should play an important role for the plastic deformation behavior [36,39-41]. According to Masing hardening, the strain hardening rate during the elasto-plastic transition stage for the heterogeneous structures can be expressed as following:

$\frac{\partial \bar{\sigma}}{\partial \bar{\varepsilon}}=E\left(1-F\left(\sigma_{i}\right)\right)$

where $\bar{\sigma}, \bar{\varepsilon}$ are equivalent stress and strain, respectively, $E$ is Young's modulus, and $F\left(\sigma_{i}\right)$ represents the hardening decay from the yielding domains (grains). As indicated from Eq. (2), the hardening rate is $E$ when there is no yielding grains (linear elastic stage), while the hardening rate decreases with increasing number of yielding grains. Moreover, the hardening rate should be strong during the elasto-plastic transition stage due to the contribution of $E$ (from the unyielding grains), which is exactly indicated in the stress strain curves of Fig. 2.

\subsection{HCP transformation and hierarchical deformation nanotwins due to low SFE}

The whole deformation microstructures for two heterogeneous grain structures at applied tensile strains of $10 \%$ and $20 \%$ are displayed in Fig. 4. It can be observed that numerous SFs, deformation twins and HCP transformations are found in the grain interiors for both heterogeneous structures. At large applied tensile strains, nearly all grains are under plastic deformation with various defects, especially it can be observed that the shapes of four big grains change dramatically after large applied tensile strains.

Fig. 5 shows the typical formation process of HCP phase by closeup views of snapshots at various applied tensile strains $(8 \%, 9 \%, 10 \%$, and $12 \%$ ) for microstructures I. As we know, an intrinsic SF are composed of two consecutive HCP atom layers (as shown in Fig. 5(a)). While, when two intrinsic SFs are initiated from one GB at adjacent slip planes (as shown in Fig. 5(a)), one narrow HCP phase can be formed when these two intrinsic SFs propagate together towards the opposite GB. The HCP phase can be thicker when three intrinsic SFs propagate together at adjacent slip planes (as shown in Fig. 5(a)). Moreover, as indicated in Fig. 5(b), one HCP phase embryo with large thickness can be formed 

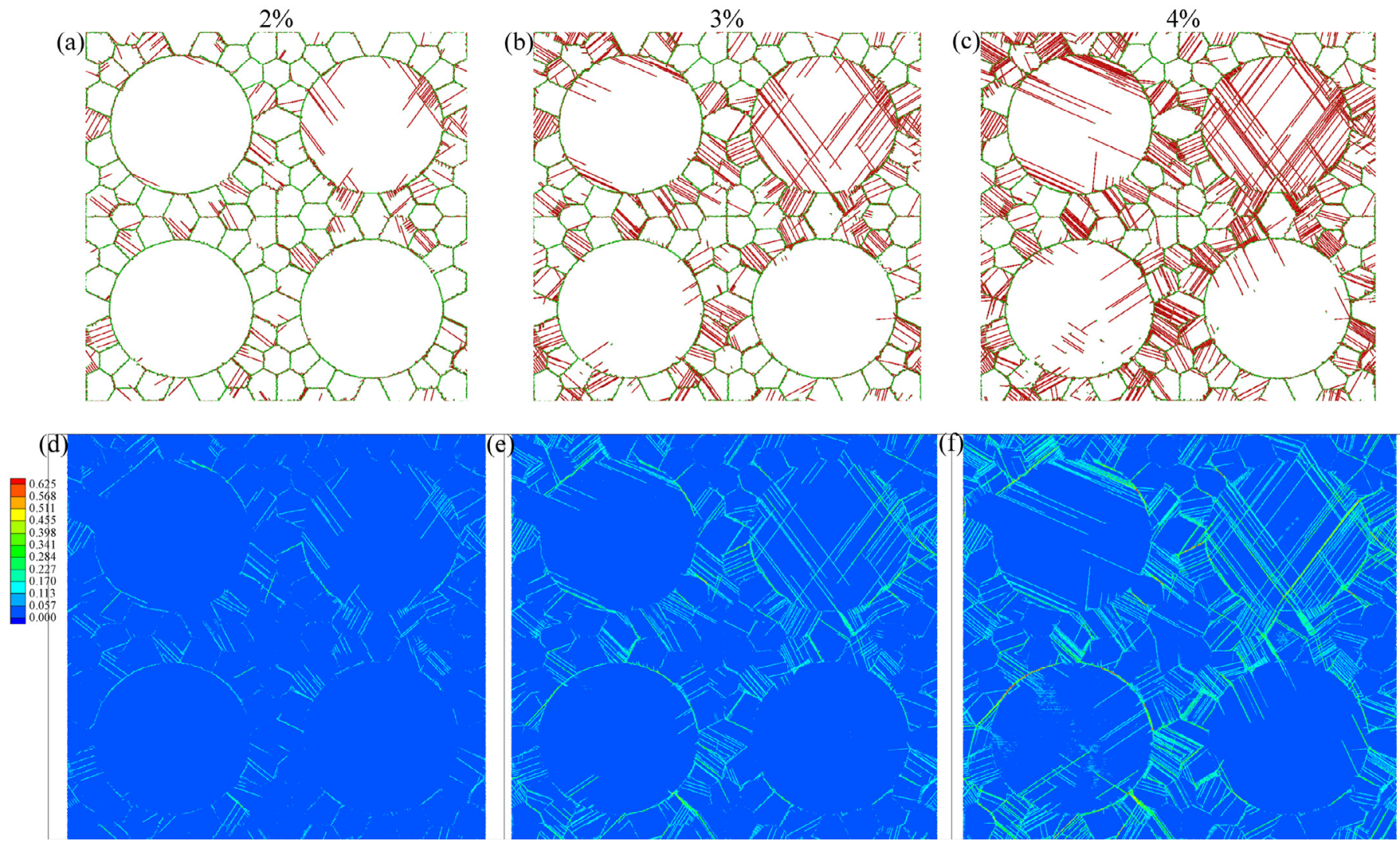

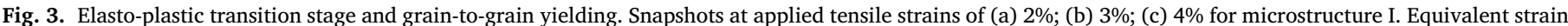
contours at applied tensile strains of (d) $2 \%$; (e) $3 \%$; (f) $4 \%$ for microstructure I.

$10 \%$

(a)

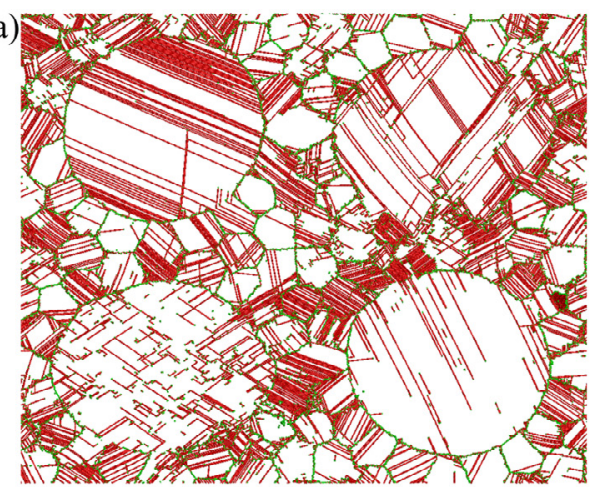

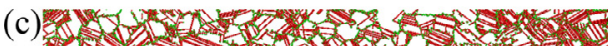

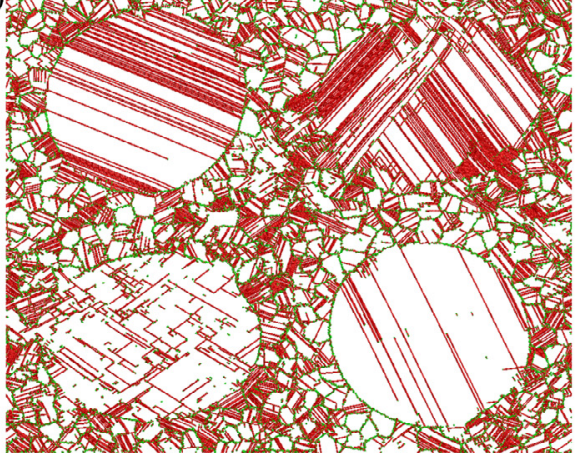

$20 \%$

(b)

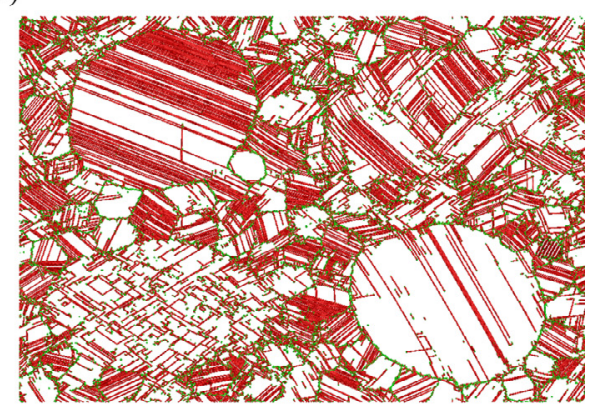

(d)

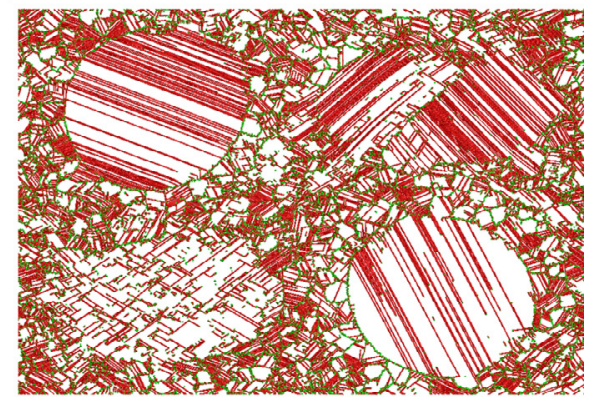

Fig. 4. The whole deformation patterns for microstructure I and microstructure II. Snapshots for microstructure I at applied tensile strains of (a) $10 \%$; (b) $20 \%$. Snapshots for microstructure II at applied tensile strains of (c) $10 \%$; (d) $20 \%$. 

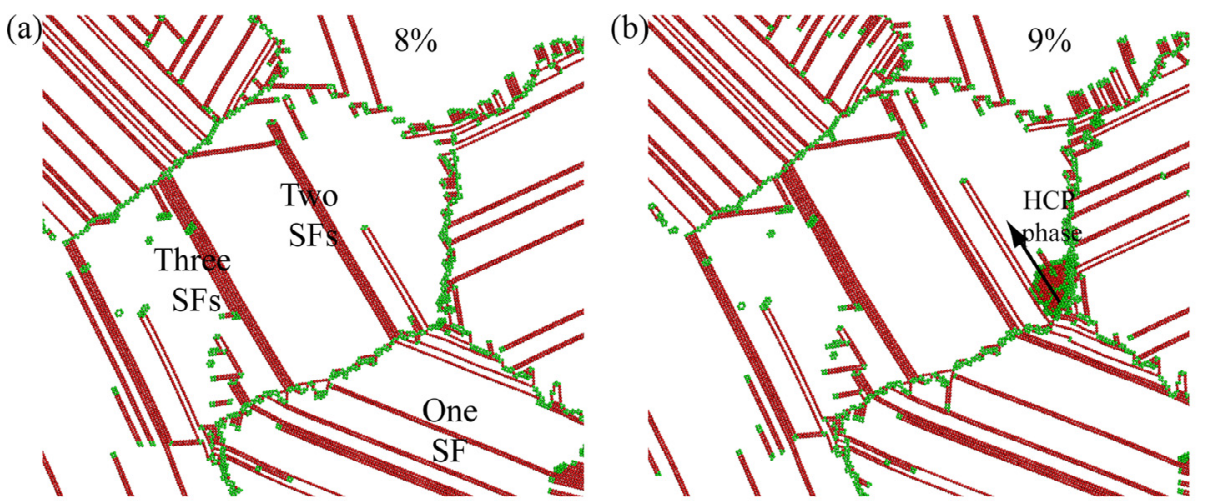

Fig. 5. Close-up views of snapshots for microstructure I at applied tensile strains of (a) $8 \%$; (b) $9 \%$; (c) $10 \%$; (d) $12 \%$ showing the HCP transformation. Perfect FCC atoms are not shown in this figure.
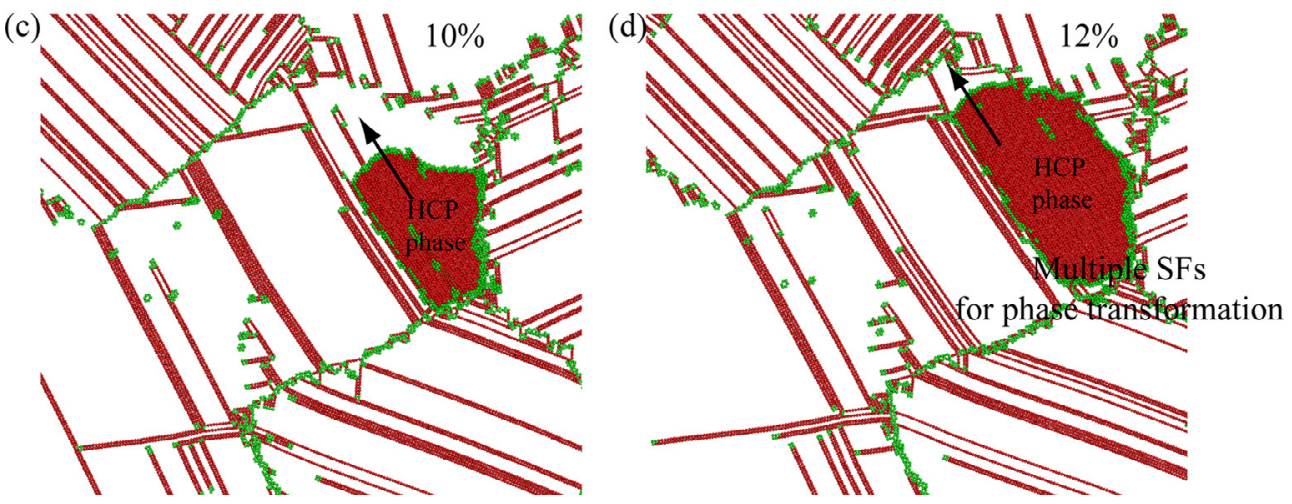

when lots of intrinsic SFs are nucleated together from the same GB at adjacent slip planes. This HCP phase embryo can grow larger and larger, and one new grain with HCP phase can be formed when these intrinsic SFs propagate together towards the opposite GB (Fig. 5(c) and (d)). Thus, the phase transformation as observed in Fig. 5 is formed by simultaneous nucleation and propagation of multiple intrinsic SFs at adjacent slip planes from the same GB.

This kind of phase transformation not only can be observed in lots of grains in microstructures I, but also in microstructures II. And the HCP phase embryo can be nucleated from GBs due to the high stress concentration at GBs or triple junctions on one hand. On the other hand, the formation of HCP phase can be attributed to the low SFE for this CrCoNi MEA at cryogenic temperature, and this SFE can even be tunable locally [31]. The similar HCP phase transformation has also been reported experimentally in the same CrCoNi MEA [26,61], in the FeMn30-Co10-Cr10 [9], and in the Co-27Cr-5Mn-0.05C HEA [62], at larger strain levels and/or at high strain rates/low deformation temperatures. The phase boundaries can be generated between the newly formed grains with HCP phase and the FCC grains in the matrix, and these phase boundaries should be effective barriers for the propagating dislocations in the FCC matrix since either $\langle c\rangle$ or $\langle c+a\rangle$ dislocations are required for transmission of edge dislocations from the FCC matrix into the HCP phase [26]. It is well known that very high critical resolved shear stress is typically required for the activation of these $\langle c\rangle$ or $\langle c+a\rangle$ dislocations, thus the mechanically-induced formation of HCP $\varepsilon$ martensite phase should be very similar to the TRIP effect as frequently reported in TRIP steels [63]. Thus, the formation of HCP $\varepsilon$-martensite phase should result in strong strain hardening and help for sustaining large tensile ductility.

Fig. 6 displays the typical multiple deformation twins (primary, secondary, and tertiary twins) by close-up views of snapshots at various applied tensile strains (10\%, and 20\%) for microstructures I. Different oriented nanotwins (primary, secondary, and tertiary twins) are marked with different colors. The atomic patterns of the localized $\{111\}$ planes in different twinning areas are marked by lines with different colors (blue, black and pink colors). The angle between the twin boundaries (TBs) of primary twins and secondary twins is approximately $70^{\circ}$. The angle between the twin boundaries (TBs) of secondary twins and tertiary twins is also approximately $70^{\circ}$. While the angle between the twin boundaries (TBs) of primary twins and tertiary twins is about $40^{\circ}$. It has been reported that hierarchical nanotwins with different orientations can further enhance the mechanical properties in metals and alloys with low SFE when compared to the single nanotwins, since multiple twins are more effective in obstructing dislocation slip and in accumulating dislocations. Thus, the structure with hierarchical nanotwins can further increase the strength limit of metals and alloys without sacrificing ductility $[64,65]$. It has been observed that HCP phase transformation and/or hierarchical nanotwins are formed in different areas of the two hierarchical grain structures. It also should be noted that the dominant deformation mechanisms of metals and alloys can be switched between phase transformation and deformation twins, or a mixture of the two mechanisms by regulating the SFE and the local stress/strain levels [63]. In our simulation cells, the local stress/strain levels are varying, and also the local SFE is tunable due to the nature of local chemical order of this MEA [31]. Thus a mixture of deformation mechanisms for HCP phase transformation and hierarchical nanotwins can be observed in this MEA with hierarchical grain structures, and these two deformation mechanisms should both result in strong strain hardening for large tensile ductility, as observed experimentally in our previous research [25-27].

\subsection{Strain partitioning between large grains and small grains}

In order to investigate the strain partitioning between large grains and small grains, the equivalent strain contours for both heterogeneous grain structures at applied tensile strains of $10 \%$ and $20 \%$ are displayed in Fig. 7(a)-(b) and (d)-(e), respectively, and the corresponding tensile strain contours are shown in Fig. 8(a) and (b) and (d) and (e), respectively. The average equivalent and tensile strains for large grains and small grains were calculated by summations of strains of atoms in 
(a)

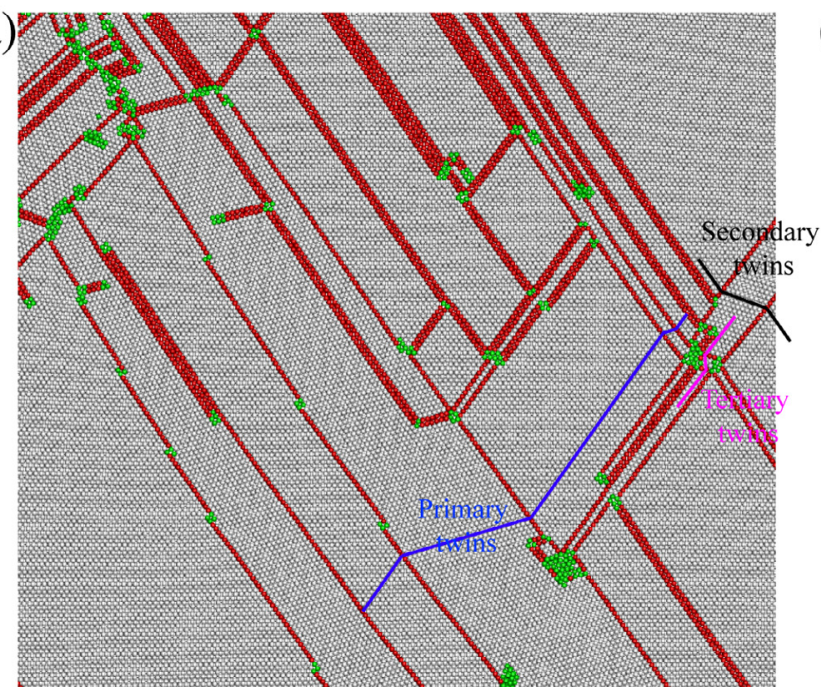

(b)

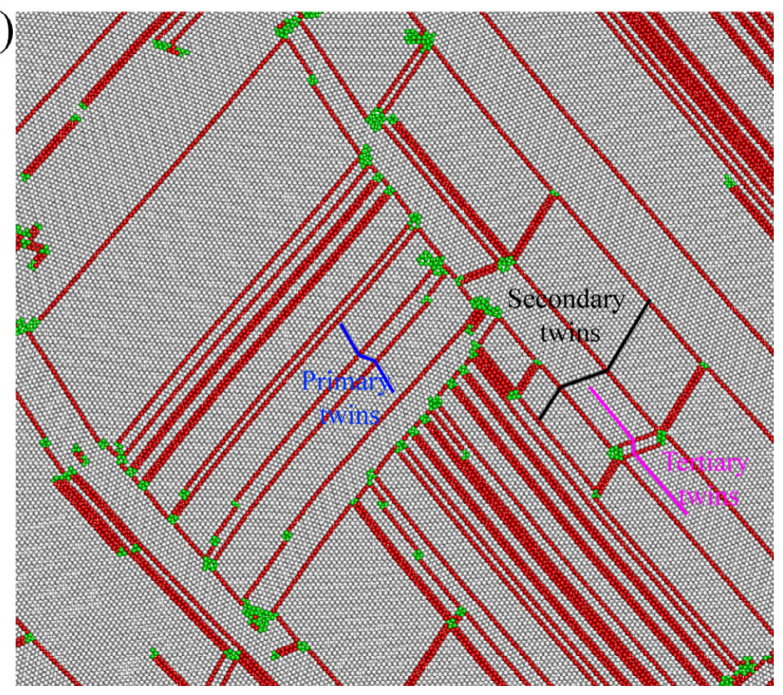

Fig. 6. Close-up views of snapshots for microstructure I at applied tensile strains of (a) 10\%; (b) $20 \%$ showing the multiple deformation twins.

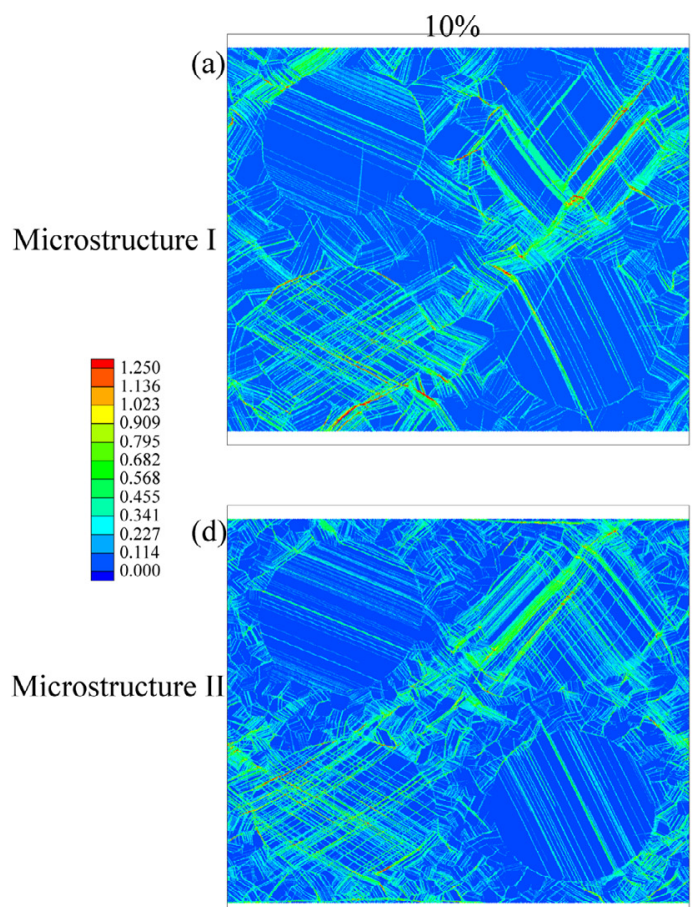

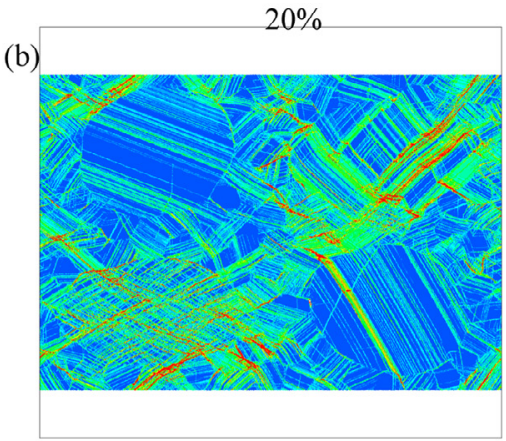

(c)

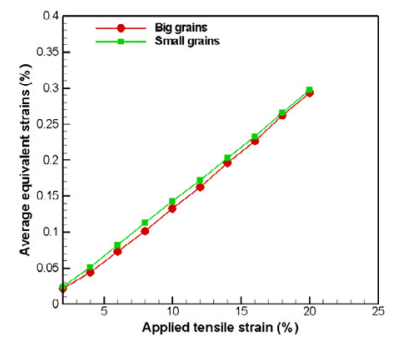

(e)

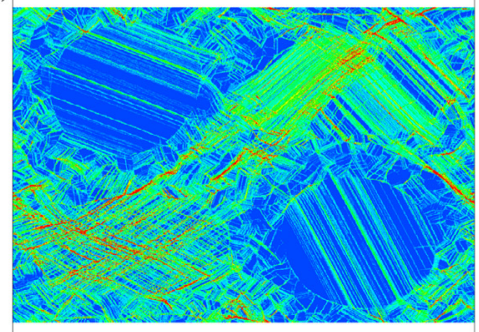

(f)

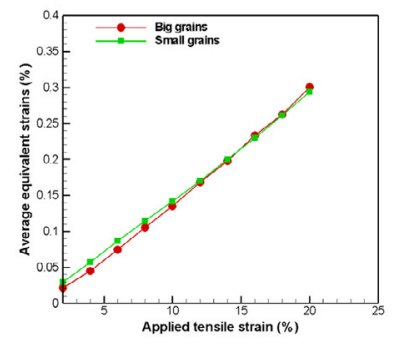

Fig. 7. Equivalent strain partitioning in two heterogeneous microstructures. Equivalent strain contours at applied tensile strains of (a) $10 \%$; (b) $20 \%$ for microstructure I. Equivalent strain contours at applied tensile strains of (d) $10 \%$; (e) $20 \%$ for microstructure II. The average equivalent strains for four big grains and the other small grains vs. the applied tensile strain for (c) microstructure I; (f) microstructure II.

large grains or small grains divided by the total atoms of large grains or small grains, respectively. Then, the average equivalent strains of large grains and small grains as a function of applied tensile strain have been plotted in Fig. 7(c) and (f) for microstructure I and microstructure II, respectively. It is clearly indicated that there is no obvious equivalent strain partitioning between large grains and small grains in the heterogeneous grain structures upon tensile straining. According to previous research [42], it is well known that the dominant deformation mechanisms can be switched from the dislocation activities to the GB activities when the grain size decreases to 10 or $20 \mathrm{~nm}$. Thus GBs of small grains, which contribute a non-ignorable part of total volume due to small grain sizes, can accommodate significant shear strains due to the GB activities. Therefore, these shear strains at GBs can compensate the lacking in equivalent strain by dislocation activities in small grains, resulting in no obvious equivalent strain partitioning between large grains and small grains (as indicated in Fig. 7(c) and (f)).

However, the tensile strain partitioning between large grains and small grains should be more important for the heterogeneous grain structures since the deformation mode is uni-axial tensile loading in the present study. The average tensile strains of large grains and small grains as a function of the applied tensile strain are plotted in Fig. 8(c) and (f) for microstructure I and microstructure II, respectively. It can clearly observed that the tensile strain partitioning between large grains and small grains in the heterogeneous grain structures exists, and this tensile strain partitioning is more obvious in microstructure II when compared to the microstructure I. Four large grains carry more average tensile 


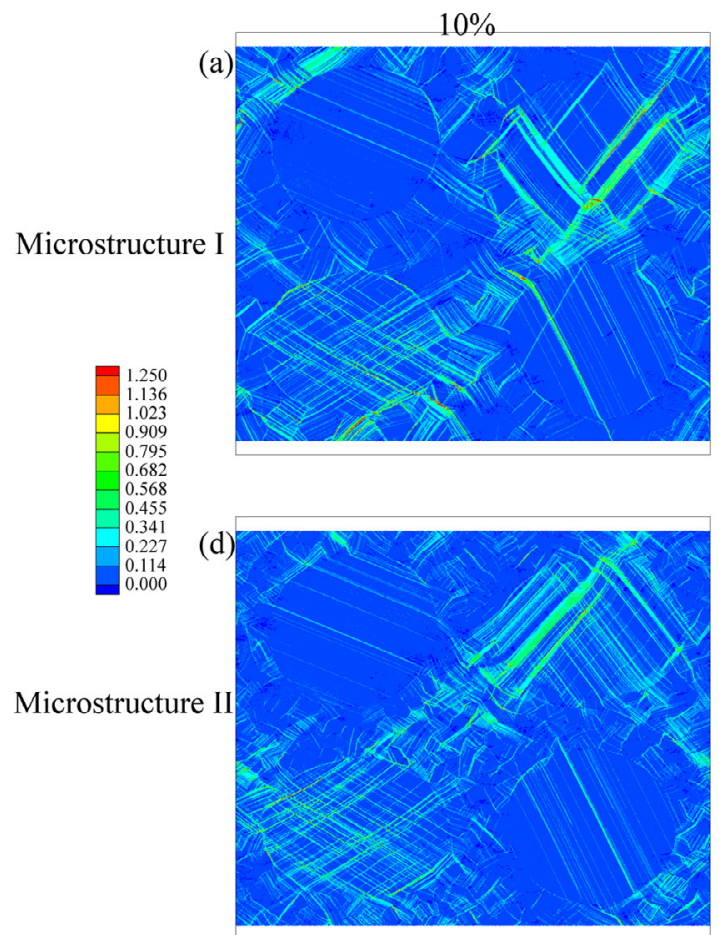

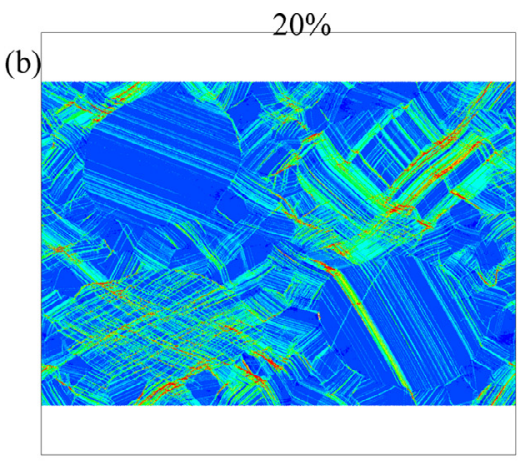

(c)

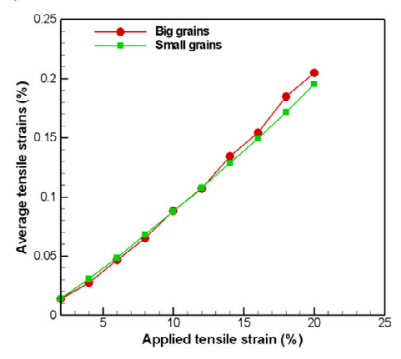

(e)

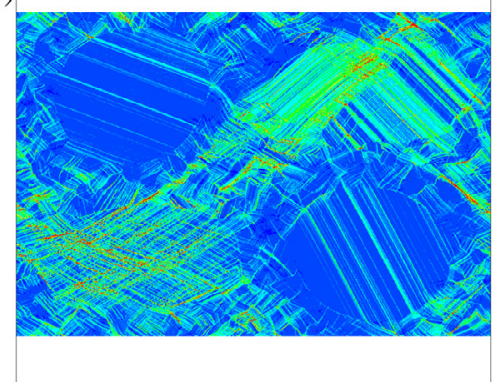

(f)

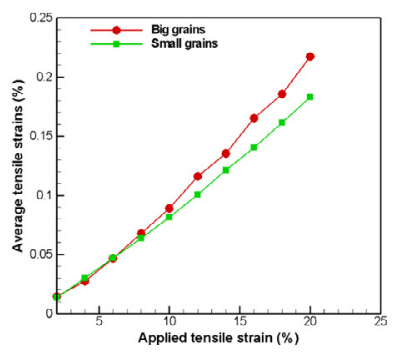

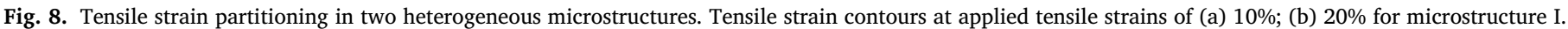

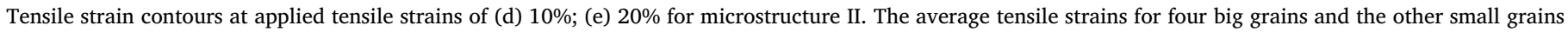
vs. applied tensile strain for (c) microstructure I; (f) microstructure II.

strains than the other small grains, resulting in tensile strain partitioning. This observation indicates that the tensile strain partitioning in the heterogeneous grain structures becomes more significant with increasing grain size ratio between four large grains and the other small grains. In our previous papers $[27,36,39]$, significant tensile strain partitioning has been found experimentally in the heterogeneous structures, and the tensile strain partitioning can be as high as $4.78\left(\bar{\varepsilon}_{\text {tensile }}^{\text {largerains }} / \bar{\varepsilon}_{\text {tensile }}^{\text {smallgrains }}\right)$ [36]. In our previous paper [27,36,39], the largest grain size for the CG is about tens of $\mu \mathrm{m}$, and the smallest grain size for the ultrafine grains (UFG) is about $100-200 \mathrm{~nm}$, thus the grain size ratio and the grain size are much larger in the experiments than those in the simulations of present study. However, the present simulated results can provide insights for the designing of heterogeneous structures and the strain partitioning mechanisms for the experiments. Thus, it is possible that both high yield strength and large tensile ductility can be achieved in heterogeneous grain structures due to the assistance of back stress developed during yielding and subsequent plastic deformation, and the back stress can be attributed to the strain partitioning between large grains and small grains, the strain gradients and the geometrically necessary dislocations (GNDs) at domain boundaries [36,39-41]. In general, the back stress can be estimated experimentally by the loading-unloadingreloading tests at quasi-static strain rate and the equations in the previous papers $[36,40]$. However, it is hard to obtain the real back stress by MD simulations due to the high strain rates in MD simulations. It might be possible to provide the "nominal back stress" at high strain rates by simulating the loading-unloading-reloading tests at various applied tensile strains in MD simulations, which would be done in the future work.

\section{Concluding remarks}

In the present study, the atomistic tensile deformation mechanisms of a CrCoNi MEA with heterogeneous grain structures have been revealed by large-scale molecular dynamics simulations. The main findings can be summarized as follows:
(1) Strong strain hardening has been observed after the linear elastic stage, and this strong strain hardening can be attributed to Masing hardening, i.e., the back stress hardening due to the grain-tograin yielding. The grain-to-grain yielding upon uni-axial tensile loading can be understood to be caused by the different Schmidt factors and the various grain sizes.

(2) The formations of HCP $\varepsilon$-martensite phase and hierarchical deformation nanotwins have been found in the two heterogeneous grain structures due to the low SFE at cryogenic temperature and the locally tunable SFE for this MEA. These two deformation mechanisms should both result in strong strain hardening for large tensile ductility, similar to the TRIP and TWIP effects in previous research $[9,26,63]$. The HCP $\varepsilon$-martensite phase was observed to be formed by simultaneous nucleation and propagation of lots of intrinsic SFs at adjacent slip planes from the same GB.

(3) No obvious equivalent strain partitioning between large grains and small grains in both heterogeneous grain structures was observed, which can be attributed to the enhanced GB activities and the accommodated shear strains at GBs in the small grains. While the tensile strain partitioning should be more important for the heterogeneous grain structures due to the uni-axial tensile loading mode in the present study. An obvious tensile strain partitioning between large grains and small grains in the heterogeneous grain structures was found, and this tensile strain partitioning was found to be more obvious with increasing grain size ratio between large grains and small grains. Due to this tensile strain partitioning, both high yield strength and large tensile ductility can possibly be achieved in the heterogeneous structures, originated from the assistance of back stress developed during yielding and subsequent plastic deformation $[25,26,35,36,38-41]$. The aforementioned deformation mechanisms should provide insights for optimizing heterogeneous structures to obtain superior mechanical properties in metals and alloys. 


\section{Declaration of Competing Interest}

The authors declared that they have no conflicts of interest to this work. We declare that we do not have any commercial or associative interest that represents a conflict of interest in connection with the work submitted.

\section{Acknowledgments}

The work was supported by the National Key R\&D Program of China [grant number 2017YFA0204402]; the National Natural Science Foundation of China [grant numbers 11672313, 11572328 and 11790293], and the Strategic Priority Research Program of the Chinese Academy of Sciences[grant number XDB22040503]. The simulations reported in the present study were performed at Supercomputing Center of Chinese Academy of Sciences.

\section{References}

[1] J.W. Yeh, S.K. Chen, S.J. Lin, J.Y. Gan, T.S. Chin, T.T. Shun, C.H. Tsau, S.Y. Chang, Nanostructured high-entropy alloys with multiple principal elements: novel alloy design concepts and outcomes, Adv. Eng. Mater. 6 (2004) 299-303.

[2] B. Cantor, I.T.H. Chang, P. Knight, A.J.B. Vincent, Microstructural development in equiatomic multicomponent alloys, Mater. Sci. Eng. A 375 (2004) 213-218.

[3] F. Otto, A. Dlouhý, C. Somsen, H. Bei, G. Eggeler, E.P. George, The influences of temperature and microstructure on the tensile properties of a CoCrFeMnNi high-entropy alloy, Acta. Mater. 61 (2013) 5743-5755.

[4] B. Gludovatz, A. Hohenwarter, D. Catoor, E.H. Chang, E.P. George, R.O. Ritchie, A fracture-resistant high-entropy alloy for cryogenic applications, Science 345 (2014) 1153-1158.

[5] Y. Zhang, T.T. Zuo, Z. Tang, M.C. Gao, K.A. Dahmen, P.K. Liaw, Z.P. Lu, Microstructures and properties of high-entropy alloys, Prog. Mater. Sci. 61 (2014) 1-93.

[6] B. Schuh, F. Mendez-Martin, B. Völker, E.P. George, H. Clemens, R. Pippan, A. Hohenwarter, Mechanical properties, microstructure and thermal stability of a nanocrystalline $\mathrm{CoCrFeMnNi}$ high-entropy alloy after severe plastic deformation, Acta. Mater. 96 (2015) 258-268.

[7] Y.F. Ye, Q. Wang, J. Lu, C.T. Liu, Y. Yang, High-entropy alloy: challenges and prospects, Mater. Today 19 (2015) 349-362.

[8] J.Y. He, H. Wang, H.L. Huang, X.D. Xu, M.W. Chen, Y. Wu, X.J. Liu, T.G. Nieh, K. An, Z.P. Lu, A precipitation-hardened high-entropy alloy with outstanding tensile properties, Acta. Mater. 102 (2016) 187-196.

[9] Z. Li, K.G. Pradeep, Y. Deng, D. Raabe, C.C. Tasan, Metastable high-entropy dual-phase alloys overcome the strength-ductility trade-off, Nature 534 (2016) $227-230$.

[10] Z.F. Lei, X.J. Liu, Y. Wu, H. Wang, S.H. Jiang, S.D. Wang, X.D. Hui, Y.D. Wu, B. Gault, P. Kontis, Enhanced strength and ductility in a high-entropy alloy via ordered oxygen complexes, Nature 563 (2018) 546-550.

[11] Y.H. Jo, S. Jung, W.M. Choi, S.S. Sohn, H.S. Kim, B.J. Lee, N.J. Kim, S. Lee, Cryogenic strength improvement by utilizing room-temperature deformation twinning in a partially recrystallized VCrMnFeCoNi high-entropy alloy, Nat. Commun. 8 (2017) 15719.

[12] T. Yang, Y.L. Zhao, Y. Tong, Z.B. Jiao, J. Wei, J.X. Cai, X.D. Han, D. Chen, A. Hu, J.J. Kai, Multicomponent intermetallic nanoparticles and superb mechanical behaviors of complex alloys, Science 362 (2018) 933-937.

[13] Y. Zhang, T.T. Zuo, Z. Tang, M.C. Gao, K.A. Dahmen, P.K. Liaw, Z.P. Lu, Microstructures and properties of high-entropy alloys, Prog. Mater. Sci. 20 (2017) 293-300.

[14] M.H. Tsai, J.W. Yeh, High-entropy alloys: a critical review, Mater Res Lett 2 (2014) $107-123$.

[15] D.B. Miracle, O.N. Senkov, A critical review of high entropy alloys and related concepts, Acta. Mater. 122 (2017) 448-511.

[16] E.P. George, D. Raabe, R.P. Ritchie, High-entropy alloys, Nat. Rev. Mater. 4 (2019) 515-534.

[17] P.J. Shi, W.L. Ren, T.X. Zheng, Z.M. Ren, X.L. Hou, J.C. Peng, P.F. Hu, Y.F. Gao, Y.B. Zhong, P.K. Liaw, Enhanced strength-ductility synergy in ultrafine-grained eutectic high-entropy alloys by inheriting microstructural lamellae, Nat. Commun. 10 (2019) 489.

[18] L.J. Santodonato, P.K. Liaw, R.R. Unocic, H. Bei, J.R. Morris, Predictive multiphase evolution in Al-containing high-entropy alloys, Nat. Commun. 9 (2018) 4520.

[19] Y.J. Liang, L.J. Wang, Y.R. Wen, B.Y. Cheng, Q.L. Wu, T.Q. Cao, Q. Xiao, Y.F. Xue, G. Sha, Y.D. Wang, High-content ductile coherent nanoprecipitates achieve ultrastrong high-entropy alloys, Nat. Commun. 9 (2018) 4063.

[20] B. Gludovatz, A. Hohenwarter, K.V. Thurston, H. Bei, Z. Wu, E.P. George, R.O. Ritchie, Exceptional damage-tolerance of a medium-entropy alloy $\mathrm{CrCoNi}$ at cryogenic temperatures, Nat. Commun. 7 (2016) 10602.

[21] Z. Wu, H. Bei, F. Otto, G.M. Pharr, E.P. George, Recovery, recrystallization, grain growth and phase stability of a family of FCC-structured multi-component equiatomic solid solution alloys, Intermetallics 46 (2014) 131-140.

[22] Z.J. Zhang, H.W. Sheng, Z.J. Wang, B. Gludovatz, Z. Zhang, E.P. George, Q. Yu, S.X. Mao, R.O. Ritchie, Dislocation mechanisms and 3D twin architectures generate exceptional strength-ductility-toughness combination in CrCoNi medium-entropy alloy, Nat. Commun. 8 (2017) 14390.
[23] Y.L. Zhao, T. Yang, Y. Tong, J. Wang, J.H. Luan, Z.B. Jiao, D. Chen, Y. Yang, A. Hu, C.T. Liu, J.J. Kai, Heterogeneous precipitation behavior and stacking-faultmediated deformation in a CoCrNi-based medium-entropy alloy, Acta. Mater. 138 (2017) 72-82.

[24] J. Miao, C.E. Slone, T.M. Smith, C. Niu, H. Bei, M. Ghazisaeidi, G.M. Pharr, M.J. Mills, The evolution of the deformation substructure in a Ni-Co-Cr equiatomic solid solution alloy, Acta. Mater. 132 (2017) 35-48.

[25] M.X. Yang, D.S. Yan, F.P. Yuan, P. Jiang, E. Ma, X.L. Wu, Dynamically reinforced heterogeneous grain structure prolongs ductility in a medium-entropy alloy with gigapascal yield strength, Proc. Natl. Acad. Sci. USA 115 (2018) 7224 7229.

[26] Y. Ma, F.P. Yuan, M.X. Yang, P. Jiang, E. Ma, X.L. Wu, Dynamic shear deformation of a CrCoNi medium-entropy alloy with heterogeneous grain structures, Acta. Mater. 148 (2018) 407-418.

[27] M.X. Yang, L.L. Zhou, C. Wang, P. Jiang, F.P. Yuan, E. Ma, X.L. Wu, High impact toughness of CoCrNi medium-entropy alloy at liquid-helium temperature, Scr. Mater. 172 (2019) 66-71.

[28] S.S. Sohn, A.K. da Silva, Y. Ikeda, F. Kormann, W.J. Lu, W.S. Choi, B. Gault, D. Ponge, J. Neugebauer, Ultrastrong medium-entropy single-phase alloys designed via severe lattice distortion, Adv. Mater. 31 (2019) 1807142.

[29] S. Yoshida, T. Bhattacharjee, Y. Bai, N. Tsuji, Friction stress and Hall-Petch relationship in CoCrNi equi-atomic medium entropy alloy processed by severe plastic deformation and subsequent annealing, Scr. Mater. 134 (2017) 33-36.

[30] C.E. Slone, J. Miao, E.P. George, M.J. Mills, Achieving ultra-high strength and ductility in equiatomic CoCrNi with partially recrystallized microstructures, Acta. Mater. 165 (2019) 496-507.

[31] J. Ding, Q. Yu, M. Asta, R.O. Ritchie, Tunable stacking fault energies by tailoring local chemical order in CoCrNi medium-entropy alloys, Proc. Natl. Acad. Sci. USA 115 (2018) 8919-8924.

[32] R. Valiev, Nanostructuring of metals by severe plastic deformation for advanced properties, Nat. Mater. 3 (2004) 511-516.

[33] M.A. Meyers, A. Mishra, D.J. Benson, Mechanical properties of nanocrystalline materials, Prog. Mater. Sci. 51 (2006) 427-556.

[34] C.C. Koch, D.G. Morris, K. Lu, A. Inoue, Ductility of nanostructured materials, MRS Bull 24 (1999) 54-58.

[35] Y. Wang, M. Chen, F. Zhou, E. Ma, High tensile ductility in a nanostructured metal, Nature 419 (2002) 912-915.

[36] X.L. Wu, M.X. Yang, F.P. Yuan, G.L. Wu, Y.J. Wei, X.X. Huang, Y.T. Zhu, Heterogeneous lamella structure unites ultrafine-grain strength with coarse-grain ductility, Proc. Natl. Acad. Sci. USA 112 (2015) 14501-14505.

[37] X.L. Wu, F.P. Yuan, M.X. Yang, P. Jiang, C.X. Zhang, L. Chen, Y.G. Wei, E. Ma, Nanodomained nickel unite nanocrystal strength with coarse-grain ductility, Sci. Rep. 5 (2015) 11728.

[38] Y.H. Zhao, T. Topping, J.F. Bingert, J.J. Thornton, A.M. Dangelewicz, Y. Li, W. Liu, Y.T. Zhu, Y.Z. Zhou, E.L. Lavernia, High tensile ductility and strength in bulk nanostructured nickel, Adv. Mater. 18 (2006) 2280-2283.

[39] Wu X.L., P. Jiang, L. Chen, F.P. Yuan, Y.T. Zhu, Extraordinary strain hardening by gradient structure, Proc. Natl Acad. Sci. USA 111 (2014) 7197-7201.

[40] M.X. Yang, Y. Pan, F.P. Yuan, Y.T. Zhu, X.L. Wu, Back stress strengthening and stain hardening in gradient structure, Mater. Res. Lett. 4 (2016) 145-151.

[41] Y.T. Zhu, X.L. Wu, Perspective on hetero-deformation induced (HDI) hardening and back stress, Mater. Res. Lett. 7 (2019) 393-398.

[42] J. Schiøtz, K.W. Jacobsen, A maximum in the strength of nanocrystalline copper, Science 301 (2003) 1357-1359.

[43] X.Y. Li, Y.J. Wei, L. Lu, K. Lu, H.J. Gao, Dislocation nucleation governed softening and maximum strength in nano-twinned metals, Nature 464 (2010) 877880.

[44] A. Sharma, P. Singh, D.D. Johnson, P.K. Liaw, G. Balasubramanian, Atomistic clustering-ordering and high-strain deformation of an $\mathrm{Al}_{0.1} \mathrm{CrCoFeNi}$ high-entropy alloy, Sci. Rep. 6 (2016) 31028.

[45] D. Choudhuri, B. Gwalani, S. Gorsse, M. Komarasamy, S.A. Mantri, S.G. Srinivasan, R.S. Mishra, R. Banerjee, Enhancing strength and strain hardenability via deformation twinning in fcc-based high entropy alloys reinforced with intermetallic compounds, Acta. Mater. 165 (2019) 420-430.

[46] J. Li, Q.H. Fang, B. Liu, Y.W. Liu, Y. Liu, Mechanical behaviors of AlCrFeCuNi high-entropy alloys under uniaxial tension via molecular dynamics simulation, RSC Adv. 6 (2016) 76409-76419.

[47] Z.N. Wang, J. Li, Q.H. Fang, B. Liu, L.Z. Zhang, Investigation into nanoscratching mechanical response of AlCrCuFeNi high-entropy alloys using atomic simulations, Appl. Sur. Sci. 416 (2017) 470-481.

[48] M. Widom, W.P. Huhn, S. Maiti, W. Steurer, Hybrid Monte Carlo/molecular dynamics simulation of a refractory metal high entropy alloy, Metall. Mater. Trans. A 45A (2014) 196-200.

[49] J. Li, Q.H. Fang, B. Liu, Y. Liu, Transformation induced softening and plasticity in high entropy alloys, Acta. Mater. 147 (2018) 35-41.

[50] J.L. Hou, Q. Li, C.B. Wu, L.M. Zheng, Atomic simulations of grain structures and deformation behaviors in nanocrystalline CoCrFeNiMn high-entropy alloy, Materials (Basel) 12 (2019) 1010.

[51] Q.-J. Li, H. Sheng, E. Ma, Strengthening in multi-principle element alloys with localchemical-order roughened dislocation pathways, Nat. Commun. 10 (2019) 3563.

[52] I.A. Alhafez, C.J. Ruestes, E.M. Bringa, H.M. Urbassek, Nanoindenation into a high-entropy alloy - an atomistic study, J. Alloys Comp 803 (2019) 618-624.

[53] W.-M. Choi, Y.H. Jo, S.S. Sohn, S. Lee, B.-J. Lee, Understanding the physical metallurgy of the CoCrFeMnNi high-entropy alloy: an atomistic simulation study, NPJ Comput. Mater. 4 (2018) UNSP 1.

[54] S.D. Feng, L. Li, K.C. Chan, L. Zhao, S.P. Pan, L.M. Wang, R.P. Liu, Tuning defor- 
mation behavior of $\mathrm{Cu} 0.5 \mathrm{CoNiCrAl}$ high-entropy alloy via cooling rate gradient: an atomistic study, Intermetallics 112 (2019) 106553.

[55] S. Plimpton, Fast parallel algorithms for short-range molecular dynamics, J. Comput. Phys. 117 (1995) 1-19.

[56] D. Farkas, Model interatomic potentials and lattice strain in a high-entropy alloy, J. Mater. Res. 33 (2018) 3218-3225.

[57] F.P. Yuan, X.L. Wu, Size effects of primary/secondary twins on the atomistic deformation mechanisms in hierarchically nanotwinned metals, J. Appl. Phys. 113 (2013) 203516.

[58] F. Shimizu, S. Ogata, J. Li, Theory of shear banding in metallic glasses and molecular dynamics calculations, Mater. Trans. 48 (2007) 2923-2927.

[59] X.M. Liu, F.P. Yuan, Y.G. Wei, Grain size effect on the hardness of nanocrystal measured by the nanosize indenter, Appl. Sur. Sci. 279 (2013) 159-166.
[60] R.P. Skelton, H.J. Maier, H.-J. Christ, The Bauschinger effect, Masing model and the Ramberg-Osgood relation for cyclic deformation in metals, Mater. Sci. Eng. A 238 (1997) 377-390.

[61] J. Mao, C.E. Slone, T.M. Smith, C. Niu, H. Bei, M. Ghazisaeidi, G.M. Pharr, M.J. Mills, The evolution of the deformation substructure in a Ni-Co-Cr equiatomic solid solution alloy, Acta. Mater. 132 (2017) 35-48.

[62] A. Mani, H.F.Lope Salinas-Rodriguez, Deformation induced fcc to hcp transformation in a Co-27Cr-5Mo-0.05C alloy, mater, Sci. Eng. A 528 (2011) 3037-3043.

[63] O. Grassel, L. Kruger, G. Frommeyer, High strength Fe-Mn-(Al, Si) trip/twip steels development - properties - application, Int. J. Plast. 16 (2000) 1391-1409.

[64] X.W. Liu, L.G. Sun, L.L. Zhu, J.B. Liu, K. Lu, J. Lu, High-order hierarchical nanotwins with superior strength and ductility, Acta. Mater. 149 (2018) 397-406.

[65] H.N. Kou, J. Lu, Y. Li, High-strength and high-ductility nanostructured and amorphous metallic materials, Adv. Mater. 26 (2014) 5518-5524. 